\title{
Relação entre escore de condição corporal e escore de locomoção em vacas leiteiras submetidas a manejo semiextensivo de alimentação
}

\author{
[Relationship between body condition score and locomotion score on dairy cows submitted to \\ semiextensive feeding management] \\ A. Barbosa, C. Pizoni, G.B. Luz, M.N. Correa, V.R. Rabassa, F.A.B. Del Pino, \\ F.P. Moraes, C.F. Martins \\ Universidade Federal de Pelotas - Pelotas, RS
}

\begin{abstract}
RESUMO
Métodos observacionais subjetivos, como escore de locomoção (EL) e escore de condição corporal (ECC), têm sido amplamente utilizados para avaliação populacional de diferentes graus de claudicação e estado nutricional em bovinos. Este estudo objetivou verificar a associação longitudinal entre o escore de condição corporal e o escore de locomoção em vacas da raça Holandesa submetidas a um regime alimentar semiextensivo. O período experimental compreendeu dois anos de observações, em uma propriedade localizada no sul do Rio Grande do Sul. O grupo experimental foi constituído por 900 vacas lactantes, que foram avaliadas trimestralmente por dinâmica ortopédica pelo EL e do estado metabólico pelo ECC. A coleta desses dados foi realizada por três veterinários treinados. A fim de se verificar a correlação entre variáveis, utilizou-se o programa SAS, por meio do método de correlação de Pearson, para determinar a associação entre as variáveis avaliadas (EL e ECC), com nível de significância de 5\%. A distribuição dos diferentes escores de locomoção durante o período foi a seguinte: $57,2 \%$ dos animais tiveram o escore de locomoção 1 , ou seja, estavam saudáveis em relação ao sistema locomotor. O restante dos animais que apresentou algum grau de dificuldade de locomoção distribuiuse da seguinte forma, segundo a intensidade: $21,6 \%$ dos animais apresentaram EL2, 15,5\% deles EL3, 3,5\% deles EL4 e 2,2\% deles EL5. Quanto ao resultado da correlação, observou-se associação negativa $(r=-0,57)$

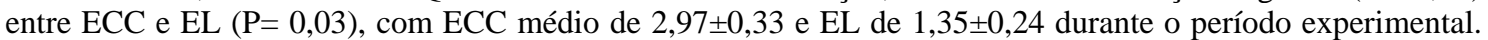
Portanto, há uma correlação negativa entre o escore de condição corporal e o escore de locomoção em vacas da raça Holandesa submetidas ao regime alimentar semiextensivo.
\end{abstract}

Palavras-chave: casco, escore, metabolismo

\begin{abstract}
Subjective observational methods such as locomotion score (LS) and body condition score (BSC) have been widely used for populations evaluation of different degrees of claudication and nutritional status in cattle. This study aimed to verify the longitudinal association between body condition score and locomotion score in Holstein cows submitted to a semi-extensive breeding regime. The experimental period comprised two years of observations at a property located in the South of Rio Grande do Sul. The experimental group consisted of 900 lactating cows, which were evaluated quarterly, being evaluated by orthopedic dynamics through LS and of the metabolic status through BCS. The collection of these data was performed by three trained veterinarians. To verify the correlation between variables, the SAS program was used using the Pearson Correlation method to determine the association between the variables evaluated (LS and BSC), at a significance level of 5\%. The rest of the animals that showed some degree of locomotion difficulty, were distributed following intensity: $21.6 \%$ of LS2 animals, $15.5 \%$ of LS3 animals, 3.5\% with LS4, and 2.2\% with LS5, while 57,2\% were without locomotion difficulty (LS1). Regarding the correlation result, a negative association ( $r=-0.57)$ was found between BSC and LS ( $P=0.03)$, with an overall of BSC of $2.97 \pm 0.33$ and $L S$ of $1,35 \pm 0.24$ during the experimental period. Therefore, there is a negative correlation between body condition score and locomotion score in Holstein cows submitted to the semi-extensive breeding regime.
\end{abstract}

Keywords: hoof, metabolism, score

Recebido em 22 de setembro de 2017

Aceito em 26 de setembro de 2017

E-mail: antoniobarbosa.vet@hotmail.com 


\section{INTRODUÇÃO}

As alterações do sistema locomotor em bovinos leiteiros vêm sendo associadas aos baixos índices de produção leiteira, o que interfere na dinâmica de produção, com reflexos negativos proporcionados ao estabelecimento rural. Talvez seja um dos maiores desafios a serem superados dentro das propriedades. As afecções podais acarretam perdas significativas, que chegam a estimar valores aproximados a 1,5kg/leite/dia por animal acometido (Warnick et al., 2001).

As perdas ligadas a afecções podais não afetam somente a produção, mas também tangem o bemestar animal, sendo responsáveis por emagrecimento progressivo e deficiência nutricional (Bicalho et al., 2009; Barbosa et al., 2016). Isso pode acarretar anestro prolongado (Barbosa et al., 2016), o que aumentaria o tempo de intervalo entre partos, com consequente impacto negativo à fertilidade. Bicalho et al. (2007) citam que o alto índice de descarte precoce é um fator que vem se agravando diante dessas perdas, com possibilidade duas vezes maior de descarte, quando se compara uma vaca claudicante com outra sem sinais álgicos ortopédicos.

Os diversos aspectos vinculados à ocorrência das afecções podais nos distintos sistemas de produção têm influenciado diretamente nos índices de acometimento ortopédico. Como exemplo, destacam-se os efeitos da sazonalidade climática ao sistema de produção semiextensivo, que, associados a características de diferentes solos, interferem na integridade física do estojo córneo (Barbosa et al., 2016). A dinâmica de locomoção dentro dos estabelecimentos rurais de criação semiextensiva é um fator limitante, aliado à ausência de preocupação com ações corretivas à aparência física da cápsula do casco, condições frequentemente observadas e negligenciadas na criação de bovinos leiteiros (Flor e Tadich, 2008).

Métodos observacionais subjetivos (escore de locomoção - EL; escore de condição corporal ECC) para a identificação e avaliação populacional de diferentes graus de claudicação e estado metabólico em bovinos vêm sendo utilizados com o intuito de diagnosticarem um estado de saúde populacional. Bicalho et al. (2009) relacionam diferentes graus de escore de locomoção com escore de condição corporal, que mostram associação positiva entre ECC com a espessura das almofadas digitais palmares e/ou plantares. De acordo com os autores, essas interferem significativamente no amortecimento biomecânico da estrutura física do estojo córneo ao solo no andamento a passo. No período de lactação, as reservas corporais são mobilizadas e canalizadas diretamente para a glândula mamária para dar suporte à produção (Rennó et al., 2011), com reflexo na perda de condição corporal e consequente impacto negativo ao sistema podal. Conforme exposto por Bicalho et al. (2009), esse fato está associado a maior ou menor grau de claudicação em rebanhos leiteiros em período de transição.

Diante de possíveis interferências do estado nutricional na dinâmica de locomoção em vacas leiteiras, este estudo teve por objetivo verificar a associação longitudinal entre escore de condição corporal e de locomoção em vacas da raça Holandesa submetidas a regime semiextensivo de criação.

\section{METODOLOGIA}

O estudo foi realizado em uma propriedade leiteira de criação de bovinos da raça Holandesa, localizada na região sul do estado do Rio Grande do Sul, na cidade de Rio Grande (latitude: 32॰15'51.46" e longitude: -52³2'21.42", com características de relevo plano e relativamente úmido. O rebanho foi criado em sistema de manejo semiextensivo.

Os animais envolvidos neste experimento foram divididos em quatro lotes, segundo produção, idade e presença de mastite, sendo submetidos a dois períodos de ordenha, com intervalo de 12 horas. A produção média foi de 23 litros/vaca/dia. Ao término da ordenha, os animais eram conduzidos à praça de alimentação, onde recebiam uma dieta com concentrado com composição segundo mostra a Tab. 1, sendo posteriormente conduzidos aos piquetes, formados por pastagem cultivada de inverno com aveia e azevém e pastagem de verão com milheto. 
Relação entre escore...

Tabela 1. Ingredientes disponíveis diariamente para as vacas leiteiras na dieta (kg) e sua composição no pré e no pós-parto

\begin{tabular}{cccccc}
\hline Alimentos & \multicolumn{3}{c}{ Pré-parto (\%) } & \multicolumn{3}{c}{ Pós-parto (\%) } \\
\hline Matéria seca & Forragem & Concentrado & Forragem & Pré-secado & Concentrado \\
FDN & 89,2 & 87,67 & 27,4 & 52,94 & 87,31 \\
FDA & 67,65 & 47,42 & 64,32 & 63,46 & 32,57 \\
Proteína total & 51,37 & 13,56 & 41,74 & 45,75 & 13,14 \\
Extrato etéreo & 9,16 & 15,61 & 9,84 & 8,88 & 14,92 \\
Minerais totais & 1,73 & 3,57 & 2,02 & 2,00 & 4,01 \\
\hline
\end{tabular}

A determinação da variável escore de condição corporal foi conduzida de acordo com método proposto por Rennó et al. (2011), em que o animal com ECC 1 era considerado severamente magro, e o animal com ECC 5 era classificado como obeso.

No decorrer de dois anos, entre 2012 e 2014, um grupo experimental de 900 vacas lactantes foi, trimestralmente, submetido à avaliação da dinâmica ortopédica por meio de escore de locomoção (EL) e de condição corporal (ECC). A coleta desses dados foi realizada por três médicos veterinários treinados. O EL variou de 1 a 5 , sendo 1 para perfeita locomoção e 5 para dificuldade de levantar o membro e andar, conforme Phillips (2010). A determinação da variável escore de condição corporal foi conduzida de acordo com método proposto por Rennó et al. (2011), em que o animal com ECC 1 era considerado severamente magro, e o animal com ECC 5 era classificado como obeso.

Todos os animais claudicantes, ou seja, que manifestavam sinais de irregularidade em sua dinâmica ortopédica, eram submetidos à avaliação clínica geral e específica do sistema locomotor.

Em relação aos resultados, para se verificar a correlação entre as variáveis, foi utilizado o programa SAS (SAS, Institute Inc. Cary, NC, EUA), por meio do método de correlação de Pearson, com o propósito de se determinar a associação entre as variáveis avaliadas (EL e ECC), em nível de significância de 5\%.
Este estudo foi aprovado (número 6243) pela Comissão de Ética em Uso de Animais da Universidade Federal de Pelotas.

\section{RESULTADOS E DISCUSSÃO}

O diagnóstico populacional da quantidade de animais claudicantes por meio do EL e do ECC tem sido de grande relevância no mapeamento diagnóstico dos fatores desencadeantes de problemas locomotores em bovinos (Bicalho et al., 2007). Desse modo, contribui para a identificação de pontos críticos e possibilita a tomada de decisões preventivas e/ou o controle, com o propósito de reduzir os efeitos negativos proporcionados à criação.

Os arranjos dos diferentes escores de locomoção durante o período se deram da seguinte forma: $69,8 \%$ dos animais apresentaram escore de locomoção 1 , ou seja, eram saudáveis no que tange ao sistema locomotor. Já os animais com algum grau de escore de locomoção se distribuíram com o restante dos animais que apresentou algum grau de dificuldade de locomoção da seguinte forma, segundo a intensidade: $21,6 \%$ dos animais apresentaram EL2, 15,5\% deles EL3, 3,5\% deles EL4, e 2,2\% deles EL5, portanto $57 \%$ dos animais encontravam-se sem nenhum tipo de dificuldade de locomoção. Esses resultados são semelhantes aos encontrados por Bicalho et al. (2007), que encontraram 11,5 (EL1), 17,3\% (EL2), 36,5\% (EL3), 37,6\% (EL4) e 1\% (EL5). Todavia, esses autores detectaram um número maior de vacas com sinais mais evidentes de claudicação, talvez pelo fato de o referido estudo ter ocorrido em vacas no sistema de free-stall, momento em que a vaca é mais desafiada, principalmente no que 
tange ao sistema locomotor, devido ao tipo de piso, mais abrasivo no free-stall, quando comparado ao sistema semiextensivo. Além disso, a taxa de animais saudáveis no estudo efetuado por Bicalho era bem menor (11,5\%) comparada à do presente trabalho. Entretanto, é importante ressaltar que a distância média percorrida diariamente pelos animais deste estudo, do campo onde se localizavam até a sala de ordenha, era de aproximadamente $2,2 \mathrm{~km}$, sendo o relevo plano, porém com alto grau de umidade, fatores esses que provavelmente contribuem negativamente para a ocorrência de afecções podais.

A distribuição dos escores de condição corporal durante o período de avaliação foi a seguinte: ECC 1 (0\%), ECC $1,5(0,3 \%)$, ECC 2 (9\%), ECC 2,5 (33\%), ECC 3 (32,8\%), ECC 3,5 (16,9\%), ECC $4(4,4 \%)$, ECC 4,5 (3,6\%) e ECC 5 (0\%). Assim, os resultados do presente estudo comprovaram a teoria discutida por Bicalho et al. (2009), pois encontrou-se associação negativa $(\mathrm{r}=-0,57)$ entre ECC e EL $(\mathrm{P}=0,03)$, com média geral de ECC de 2,97 $\pm 0,33$ e de EL de 1,35 $\pm 0,24$ durante o período experimental. Bicalho et al. (2009), ao estudarem a relação do ECC e a presença de claudicação com a espessura de coxins digitais em seu estudo desenvolvido com 700 animais, demonstraram que vacas apresentando graus de claudicação elevados apresentaram ECC e espessura de coxim digital inferiores.

O coxim tem como principal função o amortecimento da concussão do dígito ao solo; logo, quanto menor for a espessura dessa região anatômica, maior seria a predisposição a lesões que cursariam com claudicação (Bicalho et al., 2009). Portanto, conforme os dados obtidos na análise que resultou em correlação negativa para EL e ECC, pode-se sugerir que quanto menor o ECC, maiores as chances de essas vacas apresentarem claudicação. Esse resultado demonstra que a baixa condição corporal pode ser um fator que contribui para a ocorrência de possíveis danos ao sistema digital pela vulnerabilidade biomecânica de absorção do impacto das unhas ao solo. O mesmo resultado foi encontrado por Randall et al. (2015), em um estudo realizado durante oito anos em um rebanho leiteiro, o qual indica que um ECC inferior a 2 está associado com uma maior chance de claudicação em vacas leiteiras e que o risco de claudicação diminui com o aumento do ECC.

Para Buckley et al. (2003), vacas que perdem índices de condição corporal no periparto apresentam maior concentração de ácidos graxos não esterificados (AGNE), tornando-se, possivelmente, mais vulneráveis a desequilíbrios metabólicos, assim como à laminite. Esta doença, também denominada pododermatite asséptica difusa, é responsável por aproximadamente $70 \%$ das causas de claudicação em bovinos de leite e está relacionada com eventos inflamatórios e oxidativos proporcionados por esses processos (Warnick et al., 2001). O aumento nos níveis de AGNE é resultado de altas taxas de lipólise, ou seja, da mobilização de reservas lipídicas, e provoca profundo impacto no escore de condição corporal. Segundo Hoedemaker et al. (2009), existe associação entre esses fatores e o aumento do risco de claudicação. Esses autores reportaram que vacas com baixo ECC próximo ao parto tiveram de três a nove vezes mais chances do desenvolvimento de claudicação em comparação a vacas com maior ECC.

Em contrapartida ao mencionado por Buckley et al. (2003), é interessante salientar que não se pode afirmar que a associação entre emagrecimento e claudicação é um processo de causa e efeito, pois é difícil detectar as causas e as consequências, já que tanto um quanto outro podem ocorrer independentemente da ordem, bem como não se sabe se simplesmente ocorrem de forma concordante. Portanto, seria interessante um estudo analisando o início dos dois extremos: o emagrecimento e a claudicação, para, assim, compreender, de forma mais elucidativa, o mecanismo dos problemas citados, além de suas possíveis inter-relações.

Durante a lactação, as exigências energéticas são atendidas por uma combinação de nutrientes da dieta juntamente com mobilização de reservas corporais. A fase inicial da lactação é um período de grande desafio a esses animais, visto que há aumento significativo na produção leiteira e baixo consumo de matéria seca, o que resulta em um balanço energético negativo (BEN), ou seja, a ingesta alimentar não supre as necessidades metabólicas, culminando em um déficit energético, por muitas vezes fisiológico, nesse período (Rennó et al., 2011). Entretanto, há 


\section{Relação entre escore...}

momentos em que o metabolismo compensatório acaba utilizando vias alternativas para suprimento da glicose sanguínea, acontecendo a gliconeogênese. Dessa forma, não falta suprimento para o animal manter suas atividades fisiológicas, porém tal situação pode refletir-se em demasiada perda de condição corporal, bem como em comprometimento dos status metabólico, energético e imune (Drackley, 1999).

Além disso, aspectos relacionados à condição corporal e à lipomobilização com espessura de coxim digital demonstram que não só a perda de condição corporal e a consequente espessura de coxim podem estar correlacionadas com a ocorrência de afecções podais. Em estudo realizado avaliando-se o conteúdo e a composição lipídica da almofada digital de vacas e novilhas, verificou-se que a proteção do casco depende, de certa forma, da composição lipídica de ácidos graxos da almofada digital, bem como da irrigação sanguínea dele (Räber et al., 2006).

O BEN é agravado pela claudicação, pois, de acordo com Bicalho et al. (2007), vacas claudicantes têm maior dificuldade de locomoção, devido ao desconforto a que estão sendo submetidas, consequentemente não têm acesso facilitado à alimentação, o que diminui a ingestão de alimento e provoca perda de ECC. Segundo Randall et al. (2015), vacas que perdem ECC durante as primeiras quatro semanas após o parto apresentam maior fator de risco de claudicação no futuro.

No sistema de criação semiextensivo, as vacas devem ser locomovidas dos piquetes até a sala de ordenha. Conforme citaram Bicalho et al. (2007), ao serem manejadas em duas ordenhas diárias, mesmo estando claudicantes, repousam nos piquetes, com isso ingerem maior quantidade de matéria seca e chegam a produzir até 3,5 litros de leite por dia a mais do que vacas submetidas a outros sistemas de ordenha.

Ferreira (2003), ao estudar a incidência de claudicação no Brasil, realizou um ensaio com animais mantidos em sistema de confinamento (free-stall) na bacia leiteira de Minas Gerais, onde encontrou uma ocorrência anual de $122 \%$ de prevalência de lesões ortopédicas nesses rebanhos. Tal resultado demonstra que não somente $100 \%$ das vacas foram acometidas por alguma afecção podal como 22\% desses animais ainda foram reincidentes mediante episódios de claudicação durante um período de 12 meses.

Pesquisas conduzidas por Flor e Tadich (2008), com rebanhos em sistema extensivo ou sem estabulação no inverno, obtiveram uma prevalência de claudicação de 28,7\% e 32\%, respectivamente. Esses dados demonstram o benefício dos sistemas de produção não confinados para o aparelho locomotor dos animais, os quais levam a menores taxas de ocorrência de afecções podais do que os sistemas de produção confinados. Além disso, demonstraram a necessidade de investimento em tratamento e prevenção das afecções podais nos rebanhos leiteiros confinados diante dos grandes prejuízos causados.

Existem diferentes fatores que podem estar associados à ocorrência dessas desordens podais, bem como à relação entre eles. Investigam-se causas decorrentes de alteração de dieta e o reflexo desta nos status metabólico, inflamatório e fisiológico desses animais, mas ainda há escassez de informações a respeito do envolvimento de outras doenças que possam estar implicadas no desencadeamento de distúrbios ortopédicos (Lean et al., 2013). Especula-se ainda que casos de nutrição inadequada ou de doenças que provoquem quadros de anemia, levando a quadros de baixa vascularização sanguínea, vasoconstrição e possível comprometimento da circulação sanguínea podal, possam agravar, ainda mais, a hipóxia, muitas vezes citada como causa da morte das lâminas epidermais do casco (Thoefner et al., 2004).

De acordo com Rennó et al. (2011), conforme a fisiologia da lactação, esperam-se alterações fisiológicas metabólicas que relacionem a mobilização lipídica e a produção de leite ao longo de uma lactação. Essas alterações modificam a condição corporal e, consequentemente, ocorre perda de peso. Ao se avaliar o ECC e associá-lo com o EL, pode-se precocemente prevenir e diagnosticar a claudicação no rebanho, considerando-se os métodos avaliativos uma etapa fundamental para que haja redução dos impactos ao bem-estar, à produção leiteira e, principalmente para que não ocorra a desestruturação econômica e financeira 
das propriedades que adotam o sistema de manejo semiextensivo.

Com base nos efeitos deletérios de um alto ECC em bovinos, principalmente no período de transição, e na característica de correlação positiva com o amortecimento comprovada desse ECC com a diminuição de lesões ortopédicas (Bicalho et al., 2009; Buckley et al., 2003), o ECC ideal do período de transição seria em torno de 3,5 , visto que possibilitaria um amortecimento razoável, sem comprometer o estado metabólico do animal.

\section{CONCLUSÃO}

Existe uma correlação negativa entre escore de condição corporal e de locomoção em vacas da raça Holandesa submetidas ao regime semiextensivo de criação. A implantação de práticas de execução do ECC e do EL como rotina nas propriedades rurais tem o potencial de possibilitar a visualização da oscilação do status metabólico do rebanho e suas possíveis causas/consequências ao sistema locomotor de bovinos ao longo do tempo, podendo-se, assim, tomar medidas de controle e profilaxia.

\section{REFERÊNCIAS}

BARBOSA, A.B.; LUZ, G.B.; RABASSA, V.R. et al. Concentration of minerals in the hoof horny capsule of healthy and lame dairy cows. Semin. Ciênc. Agrár., v.37, p.1423-1430, 2016.

BICALHO R.C.; MACHADO, V.S.; CAIXETA, L.S. Lameness in dairy cattle: a debilitating disease or a disease of debilitated cattle? A crosssectional study of the prevalence of lameness and the thickness of the digital cushion. J. Dairy Sci., v.92, p.3175-3184, 2009.

BICALHO, R.C.; VOKEY, F.; ERB, H.N.; GUARD, C.L. Visual locomotion scoring in the first seventy days in milk: Impact on pregnancy and survival. J. Dairy Sci., v.90, p.4586-4591, 2007.

BUCKLEY, F.; O’SULLIVAN, K.; MEE, J.F. Relationships among milk yield, body condition, cow weight, and reproduction in spring-calved Holstein-Friesians. J. Dairy Sci., v.86, p.23082319, 2003.
DRACKLEY, J.K. ADSA Foundation Scholar Award. Biology of dairy cows during the transition period: the final frontier? J. Dairy Sci., v.82, p.2259-2273, 1999.

FERREIRA, P.M. Enfermidades podais em rebanho leiteiro confinado. 2003. 79f. Tese (Doutorado em Ciência Animal) - Escola de Veterinária, Universidade Federal de Minas Gerais, Belo Horizonte, MG.

FLOR, E.; TADICH, N. Claudicaciones en vacas de rebaños lecheros grandes y pequeños del sur de Chile. Arch. Med. Vet., v.40, p.125-134, 2008. HOEDEMAKER, M.; PRANGE, D.; GUNDELACH, Y. Body condition change ante- and postpartum, health and reproductive performance in German Holstein cows. Reprod. Domest. Anim., v.44, p.167-173, 2009.

LEAN, I.J.; WESTWOOD, C.T.; GOLDER, H.M.; VERMUNT, J.J. Impact of nutrition on lameness and claw health in cattle. Livest. Sci., v.156, p.71-87, 2013.

PHILLIPS, C.J. Principles of cattle production. 2.ed. Cambridge: University Press, 2010. p.75-129.

RÄBER, M.; SCHEEDER, M.R.L.; OSSENT, P. et al. The content and composition of lipids in the digital cushion of the bovine claw with respect to age and location - a preliminary report. Vet. J., v.172, p.173-1177, 2006.

RANDALL, L.V.; GREEN, M.J.; CHAGUNDA, M.G. et al. Low body condition predisposes cattle to lameness: an 8-year study of one dairy herd. J. of Dairy Sci., v.98, p.3766-3777, 2015.

RENNÓ, F.P.; BARLETTA, R.V.; JUNIOR, J.E.F. et al. Escore de condição corporal e sua relação com a produtividade, saúde e bem estar de vacas em lactação. In: SIMPÓSIO NACIONAL DE BOVINOCULTURA LEITEIRA, 3., / SIMPÓSIO INTERNACIONAL DE BOVINOCULTURA LEITEIRA, 1., 2011, Viçosa. Anais... Viçosa: [s.n.], 2011. p335-370.

THOEFNER, M.B.; POLLITT, C.C.; VAN EPS, A.W. et al. Acute bovine laminitis: a new induction model using alimentary oligofrutose overload. J. Dairy Sci., v.8, p.2932-2940, 2004.

WARNICK, L.D.; JANSSEN, D.; GUARD, C.L.; GRÖHN, Y.T. The effect of lameness on milk production in dairy cows. J. Dairy Sci., v.84, p.1988-1997, 2001. 